\title{
A full CIRCLE: inclusion of autistic doctors in the Royal College of Psychiatrists' values and Equality Action Plan
}

Sue McCowan, Sebastian C. K. Shaw, Mary Doherty, Bernadette Grosjean, Paula Blank and Malcolm Kinnear

\section{Summary}

Autistic psychiatrists bring strengths and values to the workforce and ask to be acknowledged and supported as part of the Royal College of Psychiatrists' CIRCLE values and Equality Action Plan. Courage and collaboration are required to jointly learn and innovate, promoting well-being, resilience and excellence for autistic doctors.

\section{Keywords}

Education and training; stigma and discrimination; autistic spectrum disorders; human rights; developmental disorders.

\section{Copyright and usage}

(c) The Author(s), 2022. Published by Cambridge University Press on behalf of the Royal College of Psychiatrists.
Sue Mccowan (pictured) is an old age psychiatrist in Dorset and passionate about recognition, acceptance, inclusion, support and advocacy for neurodivergent people of all ages. Sebastian Shaw is an honorary clinical lecturer (research methods and medical education) at Brighton and Sussex Medical School where he focuses on neurodiversity. Mary Doherty is a consultant anaesthetist in Ireland and founder of Autistic Doctors International, a peer-support and advocacy group for autistic doctors. Bernadette Grosjean retired as an associate professor of psychiatry from UCLA in 2016 and is currently working for a not-for-profit organisation in Los Angeles. Paula Blank is a UK consultant in child and adolescent mental health services using a pseudonym because of concerns regarding autism disclosure in the workplace. Malcolm Kinnear is a consultant in general adult psychiatry in Fife and honorary senior clinical teacher for the University of Dundee.

\section{Background}

With the recent publication of the Royal College of Psychiatrists' Equality Action Plan 2021-2023, we welcome the promotion of equality, diversity and inclusion. ${ }^{1}$ Members of the College also aspire to a values-based approach known by the acronym 'CIRCLE': Courage, Innovation, Respect, Collaboration, Learning and Excellence. Although the College welcomes the whole workforce within its Equality Action Plan and CIRCLE values, we ask for autistic doctors to be acknowledged and included, seeking collaboration regarding specific support. We are aware that other neurodivergent doctors may also wish to highlight their own needs. The College is well placed to lead the way on neurodiversity and it would seemingly be the first Medical Royal College to formally recognise and support neurodivergent doctors within policy. A diverse workforce is a well-rounded workforce and a full CIRCLE.

In surveys regarding identifying and supporting autistic patients, $1 \%$ of general practitioners and $1.1 \%$ of psychiatrists identified as autistic . ${ }^{2}$ Alongside general UK population figures of $1.1 \%$, at least 3000 UK doctors may be autistic. It is likely to be far more, however, given that medicine, and psychiatry within it, selects for many autistic strengths, ${ }^{3}$ and that non-stereotypical profiles are still underrecognised by individuals and clinicians. It seems a relatively new belief that autistic doctors even exist and can be good clinicians, supervisors and educators, yet we have been here all along doing just that, with or without the diagnostic label. In the peer-support group Autistic Doctors International (ADI), 13\% of members were psychiatrists in the last poll, second only to general practitioners. $^{3}$

\section{Autism and employment law}

Autism is already subject to employment law regarding associated disabilities but we know from peer-support groups that we, and many other neurodivergent doctors, are too often not achieving potential because of misunderstanding, stigma, underrecognition or lack of reasonable accommodations. Psychiatrists may remain cautious when considering disclosing autism, with the real risk that professional credibility may be questioned. Promoting open discussion and a culture of acceptance may encourage disclosure and better access to workplace-based adjustments, which are likely to improve well-being, resilience and retention. These are all priorities for our National Health Service workforce.

\section{Recognising strengths and attributes}

It should be noted that issues faced by autistic doctors are mostly non-clinical. Challenges more often relate to the largely non-autistic workplace set-up and to what can, at times, be confusing communication with colleagues or management. Autistic people tend to communicate better with other autistics and non-autistic people with other non-autistics. When the two neurotypes communicate with each other misunderstandings are more likely to occur, so both need to make an effort and not solely the autistic person. Most of us are not 'doctors in difficulty' and do not fulfil autistic stereotypes. This risks autistic strengths and successes going unseen, fostering a deficit-based view and facilitating the 'tragedy narrative' that too often surrounds a diagnosis. The neurodiversity paradigm fits our experience of autism as a different neurotype with many strengths, rather than pure disability or disorder, and challenges stigma, which remains pervasive throughout healthcare. The narrative we project about neurodiversity within the workforce is mirrored in our interactions with patients and their families, which can profoundly and positively influence their own perceptions and outcomes. 
Specifically acknowledging and supporting us within the Equality Action Plan also sets the expectation that we should consistently experience the College's values in the same good faith in which we practise them. Many autistic attributes are naturally aligned with these CIRCLE and core values (in italics in the paragraphs that follow).

We have demonstrated the Courage to continue as doctors despite feeling somehow 'different' from an early age. We have sustained self-belief, resilience and a growth mindset, despite the pain of our credibility and worth often being subtly or overtly challenged. We have an empathic Respect for others, often grounded in our own experiences of being outsiders. We Collaborate, finding ways to work with others to further shared goals, tenaciously Learning and Innovating as we have had to copy, analyse and then experiment with social communication since childhood, changing variables until success is achieved. We tend to 'think outside the box' and instinctively aim for Excellence as we hyperfocus on intense interests, which usually include our work. Our autistic strengths include self-motivation, curiosity, pattern recognition, attention to detail and problem-solving. ${ }^{3}$

Contrary to outdated stereotypes, many of us have increased empathy and, as such, have tended to gravitate towards the caring professions. We are aware of our position of trust and as a group have a tendency to speak out and strive for social justice - for example, unsafe patient care or staff mistreatment. Such actions are grounded in our prevailing sense of honesty and fairness. However, this comes with a risk of being misinterpreted and marginalised. As we often precisely and literally follow policies on raising issues we risk being misunderstood as trouble-making, lacking team skills and confronted for being unprofessional, without seniors considering that unspoken non-autistic rules of finding another way may not have ever been made clear.

There is an autistic tendency to value all people equally, sometimes speaking plainly and without hierarchical restriction, which should not be mistaken for lack of humility. We strive to uphold the dignity of both our autistic patients and colleagues. This can be a difficult path to tread as recognition and understanding of autism is still evolving for many professionals. However, we embrace opportunities to inform education and training with lived experience, and challenge stigma through the positive promotion of neurodiversity. In the workplace, as in life, autistic people are different, not less. We respect that our non-autistic colleagues communicate differently and ask for reciprocity regarding our different communication style.

\section{Equity}

The Foreword to the Equality Action Plan states that 'Discrimination and prejudice, based on any of the protected characteristics, is inherently wrong and can lead to profound distress and unhappiness, which negatively affects mental wellbeing. ${ }^{1} \mathrm{We}$ certainly agree with this and note that 'camouflaging' or 'masking' (hiding autistic features) is associated with increased anxiety, depression and suicidality. ${ }^{4}$ With high rates of burnout, mental illness and suicide in both doctors and autistic adults, the consideration and support of hidden autistic doctors is vital. For those of us who managed to facilitate accommodations without knowing why, this has typically been at our own expense, often accompanied by unnecessary shame and low self-esteem, alongside a potential adverse impact on health and earnings. Specialist assessment may be helpful to recommend a range of adjustments that we cannot always identify ourselves. Equity is a better approach than equality, as different people need different supports. We do not all need ear plugs, for example. However, some may not manage a working day without them.

\section{Support and signposting}

Undiagnosed and unsupported colleagues may sometimes present as 'doctors in difficulty'. It is, therefore, worth considering whether students and doctors with a variety of challenges might be unknowingly autistic. These might not be well-recognised autistic challenges but may include areas such as unfulfilled potential, spikey profiles (a large differential between strengths and challenge areas), communication issues with colleagues, time management (which could instead be transition issues), a perception of not being 'flexible', not sustaining work without repeated absences, and not benefitting as much as expected from the usual support offered. As supervisors or mentors, the College asks us to help colleagues to fulfil their full potential in carrying out their clinical and other responsibilities at work. Many are already on the way to achieving some of this naturally, in a person-centred manner, without realising that they are interacting with autistic doctors. However, understanding of autism and consistency in approach is needed for all autistic doctors. While respecting differences, appropriately experienced support should be sought, including signposting to peer support, such as that offered through ADI. ${ }^{3}$

Perceptions that autistic doctors are automatically unsuited to their careers still occur in various specialities, alongside adverse feedback without relevant help or workable, specific suggestions. One member who disclosed was simply told in writing that autism is incompatible with being a doctor. ${ }^{5}$ Support from ADI, such as letters or attending fitness to practice meetings, has changed outcomes by informing, explaining and challenging outof-date beliefs. Once implemented, we have seen examples within ADI of autistic doctors continuing their careers and thriving. The real risk of losing one's credibility and job is why many experienced and successful autistic doctors may not seek or disclose a diagnosis and fear requesting reasonable accommodations. One of our authors is using a pseudonym for these reasons. It is therefore vital that we are specifically included in the Equality Action Plan.

\section{Conclusions}

The Royal College of Psychiatrists continues to champion equality, diversity and inclusion and is well placed to lead the way with neurodiversity. A CIRCLE of values needs to encompass its whole workforce and we ask for autistic doctors to be acknowledged, valued and supported. We welcome discussion and collaboration, and trust that psychiatrists and the College are more than up to the task in this great speciality in which we are all privileged to practise.

Sue Mccowan (D), Dorset Healthcare University Foundation Trust, UK; Sebastian

C. K. Shaw, Department of Medical Education, Brighton and Sussex Medical School, UK;

Mary Doherty, Navan Hospital, Ireland; Bernadette Grosjean, University of California,

Los Angeles, USA; Paula Blank, (pseudonym), CAMHS, UK; Malcolm Kinnear,

University of Dundee, UK

Correspondence: Sue McCowan. Email: s.mccowan@nhs.net

First received 9 Jul 2021, final revision 02 Dec 2021, accepted 21 Dec 2021

\section{Author contributions}

S.M., S.C.K.S., M.D., B.J., P.B. and M.K. were responsible for the conception and design. S.M., S.C.K.S., M.D., B.J., P.B. and M.K. drafted the manuscript. S.M. S.C.K.S., M.D., B.J., P.B. and M.K. contributed to the refining of the manuscript. S.M., S.C.K.S., M.D., B.J., P.B. and M.K. gave final approval for submission. 


\section{Funding}

This research received no specific grant from any funding agency, commercial or not-for-profit sectors.

\section{Declaration of interest}

All authors are members of the peer-support and advocacy group Autistic Doctors International.

\section{References}

1 Royal College of Psychiatrists. RCPsych Equality Action Plan 2021-23. Royal College of Psychiatrists, 2021 (https://www.rcpsych.ac.uk/docs/default- source/about-us/equality-diversity-and-inclusivity/equality-action-planjanuary-2021.pdf?sfvrsn=af74a81a_6).

2 Crane L, Davidson I, Prosser R, Pellicano E. Understanding psychiatrists' knowledge, attitudes and experiences in identifying and supporting their patients on the autism spectrum: online survey. BJPsych Open 2019; 5: E33.

3 McCowan S, Shaw SCK, Doherty M, Grosjean B, Blank P, Kinnear M. Vive La difference! celebrating and supporting autistic psychiatrists with autistic doctors international. BJPsych Open 2021; 7 (S1): S40-S40.

4 Cook J, Hull L, Crane L, Mandy W. Camouflaging in autism: a systematic review. Clin Psychol Rev 2021; 89: 102080.

5 Doherty M, Johnson M, Buckley C. Supporting autistic doctors in primary care: challenging the myths and perceptions. Br J Gen Pract 2021; 71: 294-5.

\section{Reflection}

\section{Aaron Temkin Beck - an appreciation}

\section{Jan Scott $(\mathbb{D}$}

Aaron Temkin Beck (known to friends and colleagues as Tim) was born in Providence, Rhode Island, on 18 July 1921, the youngest of four children of Russian Jewish immigrants. Beck celebrated his centenary with a Zoom birthday gathering, during which he received a message from the Dalai Lama, who told Beck that 'you have lived a truly meaningful life and continue to be an inspiration to us all'. Sadly, Beck died on 1 November 2021. Although much has been and will be written about Tim, this brief article offers an appreciation of one of the world's leading researchers in psychopathology and the 'father of cognitive-behavioural therapy'.

It is sometimes forgotten that, after obtaining a medical degree from Yale in the years just after the Second World War, Beck was considering a career in neurology (attracted by the high degree of precision of that discipline). However, after a placement in psychiatry, he became fascinated with psychoanalysis. Unlike his peers, Tim didn't just practise psychoanalysis, he researched it. He began a series of carefully conceived studies that aimed to validate Freudian psychoanalytic constructs but ultimately led to the development of a new theory of depression. Like Kraepelin, Beck was an acute observer of mental states, but like Jaspers he placed great value on understanding the cognitive and emotional content of psychopathology from the patient's perspective. His critical contribution was discerning that the way we feel and behave is understandable in the context of consciously accessible beliefs. Beck successfully translated his understanding of the psychological impact of cognitive distortions into a practical therapy for depression that combined cognitive restructuring with behavioural interventions. This revolutionised therapy in the post-war decades and has continued to ensure that CBT therapists remain focused on a patient's most pressing emotional concerns. As cognitive distortions and dysfunctional beliefs are discoverable by individuals and therapists, working together allows them to collaborate in formulating ways forward. Furthermore, Beck's commitment to empirical evaluation of theoretical models and to testing therapies in clinical trials ensured that the entire field of psychotherapy has been driven to adopting a more scientific approach.

Beck's template for CBT has been adapted and extended to an extraordinary range of conditions and his approach has influenced a number of independent research groups working on depression, all anxiety disorders, eating disorders, borderline personality disorder and psychosis. Given the growth and apparent popularity of cognitive therapy and CBT, the approaches have been closely scrutinised by the scientific community. Although some criticisms are apposite, there needs to be a greater recognition that Beck always viewed his theories as provisional only and insisted on subjecting all his ideas to rigorous testing. This philosophy extended to his many other significant research contributions, ranging from his work in suicidology to the development of the Beck Depression Inventory. No other psychiatrist or psychologist of Beck's generation has had so great an impact on psychotherapy and psychological approaches to treatment. He has been identified as one of the five most influential psychotherapists of all time and has been credited with helping to shape the face of modern psychiatry. However, for many, an abiding memory of Beck will be his generosity towards researchers and therapists and the way he encouraged, trained and inspired younger colleagues, including many from the UK.

In the 1970s, Beck asked: 'Can a fledgling psychotherapy challenge the giants in the field - psychoanalysis and behaviour therapy?' The answer of course is a resounding yes. Tim's work has sent out an unforgettable signal, and the introduction of CBT will rank as a milestone in the history of 20th-century medicine.

(c) The Author(s), 2022. Published by Cambridge University Press on behalf of the Royal College of Psychiatrists 